\title{
Idiopathic Postmenopausal Uterine Inversion: A Case Report
}

\author{
Ehab Al-Rayyan ${ }^{\mathrm{a}}$, Susan Thabet ${ }^{\mathrm{a}}$, b, Mitri Rashed ${ }^{\mathrm{a}}$, Amer Gharaibeh ${ }^{\mathrm{a}}$, \\ Rami Al-Shwiyat ${ }^{\text {a }}$, Sa'ed Haddad ${ }^{\text {a }}$, Maram Almaghariz ${ }^{a}$, \\ Maher Maaita $^{a}$
}

\begin{abstract}
Inversion of the uterus is a rare gynecological condition. The majority of reported cases were encountered in the immediate postpartum period. A non-puerperal inversion occurs when the uterus acts to expel a submucous lesion attached to the fundus, such as uterine fibroid, endometrial carcinoma, sarcoma or polyp. We report a case of spontaneous postmenopausal uterine inversion without underlying pathology. The diagnosis of non-puerperal uterine inversion is often difficult and requires a high index of suspicion
\end{abstract}

Keywords: Spontaneous uterine inversion; Idiopathic uterine inversion; Postmenopausal uterine inversion; Haultain procedure

\section{Introduction}

Inversion of the uterus is a rare gynecological condition. The majority of reported cases were encountered in the immediate postpartum period [1-3]. A non-puerperal inversion occurs when the uterus acts to expel a submucous lesion attached to the fundus, such as uterine fibroid, endometrial carcinoma, sarcoma or polyp [4-5].

We report a case of spontaneous postmenopausal uterine inversion without underlying pathology.

The diagnosis of non-puerperal uterine inversion is often difficult and requires a high index of suspicion.

\section{Case Report}

A 67 -year-old woman, para $8+0$, all by normal vaginal deliveries, presented with a 2-day history of lower abdominal pain

Manuscript submitted April 3, 2019, accepted April 12, 2019

aJordanian Royal Medical Services, King Hussein Medical Centre, Amman, Jordan

${ }^{\mathrm{b}}$ Corresponding Author: Susan Thabet, Jordanian Royal Medical Services, King Hussein Medical Centre, Amman, Jordan.

Email: Theflower123@yahoo.com

doi: https://doi.org/10.14740/jmc3290 and inability to pass urine. This was associated with a mass protruding from the vagina and offensive vaginal discharge. She had no bowel symptoms.

On examination, her vital signs were normal. Abdominal examination revealed a tender palpable suprapubic mass midway between the pubic symphysis and umbilicus. Pelvic examination showed an infected fleshy, friable mass that was protruding through the introitus past the hymen with foul smelling discharge on its surface; there was no active bleeding. The acute retention was relieved with immediate urethral catheterization and $2,000 \mathrm{~mL}$ of urine was drained.

Her complete blood count, serum electrolytes and kidney function test were all within normal limits; urine analysis suggested a urinary tract infection.

The patient was admitted and started on intravenous broad spectrum antibiotics and perineal care. Examination under anesthesia showed the mass to be continuous with the cervix which could not be well delineated on palpation. On rectal examination, neither the uterus nor the mass could be felt. A biopsy was taken from the mass revealing endometrial tissue with no evidence of malignancy.

Pelvic magnetic resonance imaging (MRI) showed a Ushaped uterine cavity with thickened and inverted uterine fundus on sagittal and coronal sections (Fig. 1).

Based on the mentioned clinical and imaging findings, non-puerperal uterine inversion with super infection was strongly suspected, and the decision for hysterectomy was made and discussed with the patient.

A laparotomy was carried out, the round and utero-ovarian ligaments and part of the urinary bladder were dragged into a cup-shaped depression (Fig. 2). The constricting ring was too tight, therefore, after identifying both ureters the uterus was repositioned using the Haultain procedure followed by abdominal hysterectomy and bilateral salpingo-oophorectomy (Fig. 3). The histopathological report revealed no abnormality to which the uterine inversion could be attributed.

\section{Discussion}

Uterine inversion is defined as a decent of the uterine fundus to or through the cervix, so that the uterus is turned inside out [5].

It can be acute or chronic. Acute inversion causes severe pain and hemorrhage, and almost always is a consequence of a delivery; whereas chronic inversion is insidious and charac- 


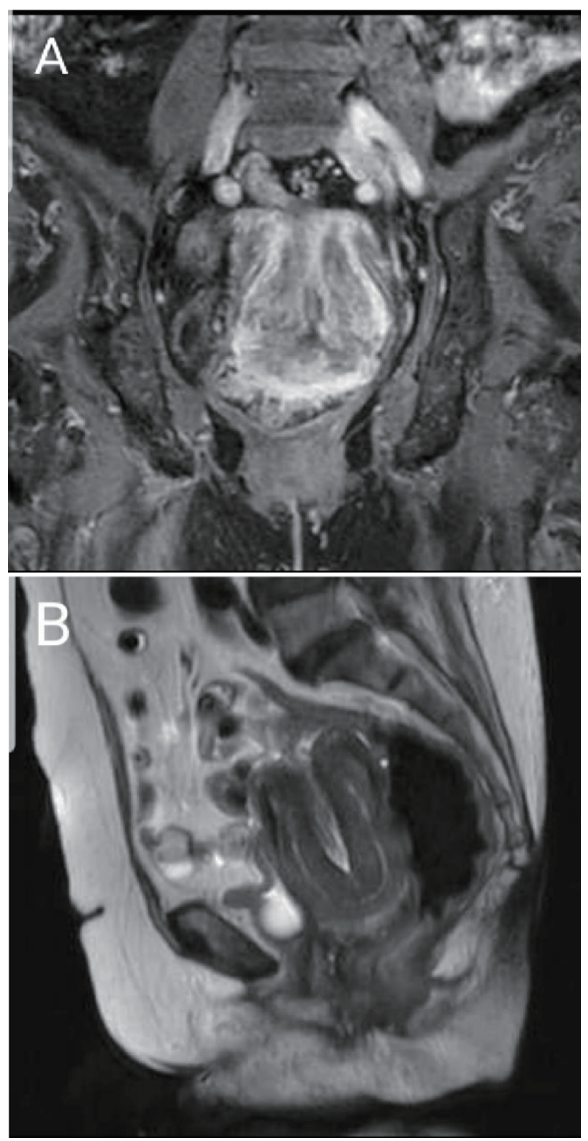

Figure 1. Coronal (A) and sagittal (B) MRI views showing U-shaped uterine cavity with thickened and inverted uterine fundus.

terized by pelvic pain, bleeding, anemia and urinary dysfunction, including urine retention, as in this case, resulting from anatomical distortion causing bladder neck obstruction [6-8].

The diagnosis is based on a high index of suspicion. Finding a mass coming through the cervix without definite cervical margins, absence of the uterine fundus or fundal dimpling during bimanual or rectal examinations suggest the diagnosis. The openings of the Fallopian tubes may be identified if it had been dragged through the endometrial surface. The diagnosis is easier with complete uterine inversion when a fleshy mass

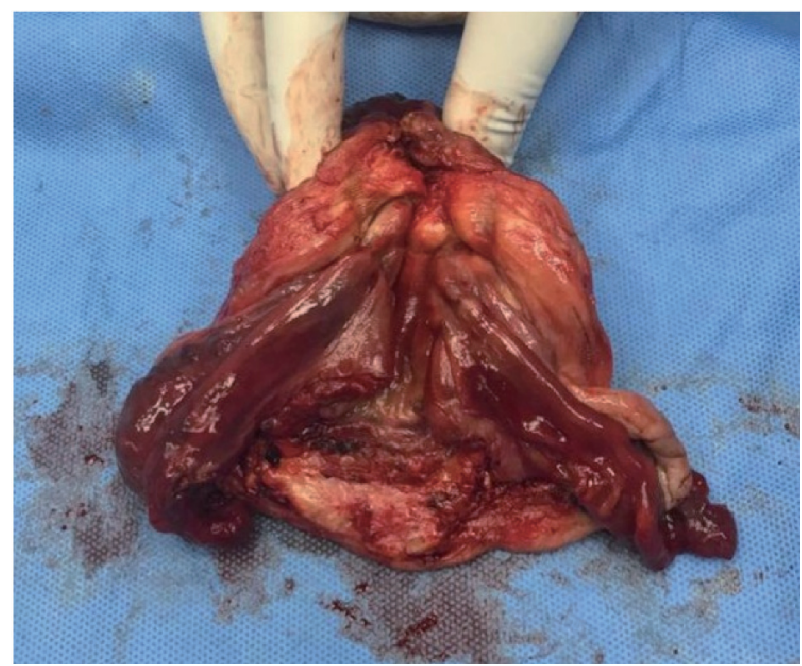

Figure 3. The removed uterus, fallopian tubes and ovaries.

protrudes from the vulva with a constricting ring of the cervix superiorly [7].

Ultrasound, computed tomography (CT) scan and MRI can help in the diagnosis of uterine inversion. The suggestive features on ultrasound include $\mathrm{Y}$ shape fundal indentation and depressed longitudinal groove extending from the uterus to the center of the inverted portion [8].

MRI is the best radiological modality not only for the correct diagnosis, but also clearly defines the lesion and its relation to neighbouring structures [9]. The features of the standard pelvic MRI protocol with T2-weighted scans will include U-shaped uterine cavity, thickened and inverted uterine fundus on sagittal section and bull's eye configuration on an axial image [7].

In most cases, treatment is guided by whether the condition is acute or chronic, stage of inversion, the reproductive wish of the patient and the cause of the inversion (benign or malignant).

In stage 1, inversion is intrauterine (incomplete) and the fundus remains within the cavity; stage 2, complete inversion of the uterine fundus through the fibromuscular cervix; stage 3 total inversion where the fundus protrudes through the vulva; stage 4, the vagina is also involved within total inversion [10].

Surgical repositioning or hysterectomy could be done

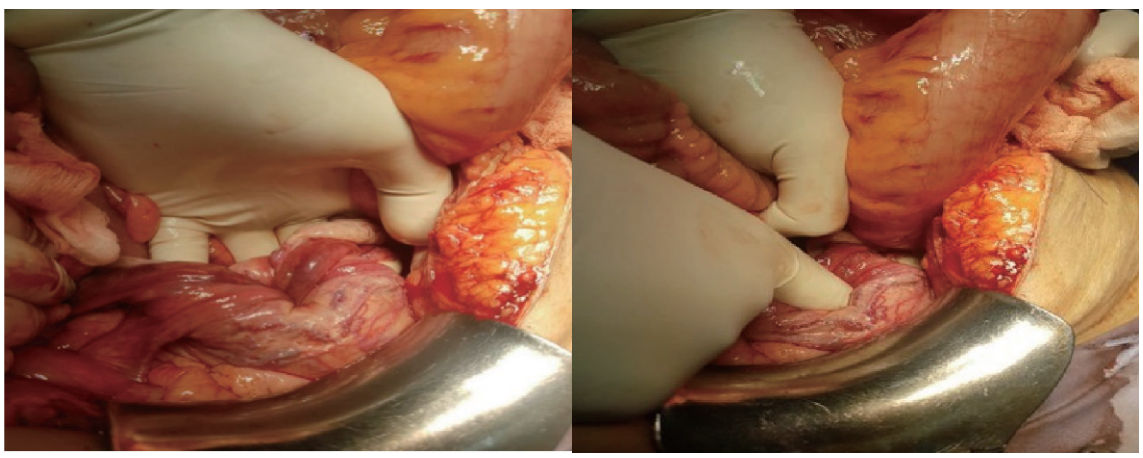

Figure 2. Uterine inversion found at laparotomy. Note the characteristic dimpling and in-turning of the round ligaments and fallopian tubes. 
through either abdominal or vaginal approach.

Spinelli and Kustner are transvaginal surgical repositioning techniques with the basic difference being that Spinelli's approach is anterior and requires dissection of the bladder and an anterior uterine wall incision; while Kustner's is a posterior approach which involves entering the space of Douglas vaginally and splitting the posterior aspect of the uterus and the cervix, which makes it a bit easier and safer [8].

Surgical repositioning can also be done through a laparotomy using the Huntington procedure, which involves a gentle upward traction of the round ligaments of the uterus after dilating the cervical ring digitally until the uterus is reinverted $[2,3,8]$.

The operation in this case is consistent with the Haultain procedure, which uses an abdominal approach in which a vertical incision in the posterior portion of the constricting cervical ring is made with traction on the round ligaments until the uterus is reinverted to its normal anatomy. This was followed by hysterectomy and bilateral salpingo-oophorectomy.

\section{Conclusions}

This case shows that chronic uterine inversion could occur without the usual predisposing factors and high index of suspicion is necessary to detect these unusual presentations.

\section{Acknowledgments}

None to declare.

\section{Financial Disclosure}

None to declare.

\section{Conflict of Interest}

Not applicable.

\section{Informed Consent}

Informed consent was obtained from the patient; publication was permitted by the center ethical committee.

\section{Author Contributions}

Each author contributed in searching for and editing the content.

\section{References}

1. de Vries M, Perquin DA. Non-puerperal uterine inversion due to submucous myoma in a young woman: a case report. J Med Case Rep. 2010;4:21.

2. Gomez-Lobo V, Burch W, Khanna PC. Nonpuerperal uterine inversion associated with an immature teratoma of the uterus in an adolescent. Obstet Gynecol. 2007;110(2 Pt 2):491-493.

3. Kilpatrick CC, Chohan L, Maier RC. Chronic nonpuerperal uterine inversion and necrosis: a case report. J Med Case Rep. 2010;4:381.

4. Gowri V. Uterine inversion and corpus malignancies: a historical review. Obstet Gynecol Surv. 2000;55(11):703707.

5. Lai FM, Tseng P, Yeo SH, Tsakok FH. Non-puerperal uterine inversion - a case report. Singapore Med J. 1993;34(5):466-468.

6. Krissi H, Peled Y, Efrat Z, Goldshmit C. Ultrasound diagnosis and comprehensive surgical treatment of complete non-puerperal uterine inversion. Arch Gynecol Obstet. 2011;283(Suppl 1):111-114.

7. Occhionero M, Restaino G, Ciuffreda M, Carbone A, Sallustio G, Ferrandina G. Uterine inversion in association with uterine sarcoma: a case report with MRI findings and review of the literature. Gynecol Obstet Invest. 2012;73(3):260-264.

8. Buyukkurt S, Vardar MA, Zeren H, Ozgunen FT. Nonpuerperal inversion of the uterus caused by leiomyosarcoma: a case report and clinical management. J Obstet Gynaecol Res. 2007;33(3):402-406.

9. Moulding F, Hawnaur JM. MRI of non-puerperal uterine inversion due to endometrial carcinoma. Clin Radiol. 2004;59(6):534-537.

10. Spinelli PG. Inversion of the uterus. Riv Ginec Comtemp. 1897;11:567-570. 